\title{
Case: Wernicke Encephalopathy after Bariatric Surgery
}

\section{Kristl Dobbelaere* and Stefaan De Clercq}

AZ ST Jozef, Oude Liersebaan 4, B-2980 Malle, Belgium

*Corresponding author: Kristl Dobbelaere, AZ ST Jozef, Oude Liersebaan 4, B-2980 Malle, Belgium, Tel: $003233802119 / 0032497240474$; E-mail: Kristl.Dobb@gmail.com

Rec date: Dec 09, 2014, Acc date: Dec 26, 2014, Pub date: Dec 28, 2014

Copyright: (c 2014 Dobbelaere K, et al. This is an open-access article distributed under the terms of the Creative Commons Attribution License, which permits unrestricted use, distribution, and reproduction in any medium, provided the original author and source are credited.

\begin{abstract}
Background: Wernicke encephalopathy after bariatric surgery is rare and difficult to recognize. The severity of the disease and the preventable but irreversible long-term implications lead us to emphasize the importance of timely and correct diagnosis and treatment. Nonalcoholic Wernicke encephalopathy often manifests atypical clinical presentation and disease progression that makes it prone to be underestimated.

Clinical case: We report the medical history of a 48-year old lady after bariatric surgery. It is a case of possible Wernicke encephalopathy. The patient presents with a constellation of physical features that may represent a complex clinical situation due to longer existing vision problems or an as yet uncharacterized Wernicke encephalopathy. We discuss diagnosis, underlying pathology, metabolic mechanism and common therapeutic approach.
\end{abstract}

Conclusion: With the ongoing global epidemic of obesity the need for obesity surgery will increase, thus leading to increased occurrence of Wernicke encephalopathy. This emphasizes the importance of awareness of this clinical entity and the need for early detection and prevention.

Keywords: Wernicke encephalopathy; Bariatric surgery; Thiaminedeficiency

\section{Introduction}

Wernicke encephalopathy after bariatric surgery is rare and difficult to recognize. The severity of the disease and the preventable but irreversible long-term implications lead us to emphasize the importance of timely and correct diagnosis and treatment. Nonalcoholic Wernicke encephalopathy often manifests atypical clinical presentation and disease progression that makes it prone to be underestimated.

We report a case of possible Wernicke encephalopathy after bariatric surgery. The patient presents with a constellation of physical features that may represent a complex clinical situation due to longer existing vision problems or an as yet uncharacterized Wernicke encephalopathy.

\section{Case Report}

A 48-year old lady presents for follow-up after bariatric surgery. She underwent a Roux-en Y gastric bypass (RYGB) 12 weeks before. Her medical background includes hypertension, breast correction, sterilization, mild OSAS and congenital retinitis pigmentosa. She is on regular medication and takes Co-diovane (valsartan $160 \mathrm{mg}$ and hydrochlorothiazide $12.5 \mathrm{mg}$ ) and acetylsalicylic acid $100 \mathrm{mg}$.

The patient reports feeling well. She has lost a lot of weight, as expected after the surgery. She has a weak appetite. On the other hand, worried family members reveal that she is often dizzy, she walks with difficulty and feels instable. The patient also has periods of memoryand vision-problems and regular episodes of vomiting.
At first observation the patient demonstrates a bilateral ptosis, which is explained by long-term vision problems due to congenital retinitis pigmentosa. Neurologic examination reveals a truncal ataxia, but no confused state, no oculomotor deficits, no horizontal gazeevoked nystagmus.

Routine blood examination was without significant abnormality.

\section{Discussion}

The patient presented for routine follow-up with no specific subjective complaints. Hetero-anamnesis and clinical examination prompted us to consider a diagnosis of Wernicke encephalopathy.

Wernicke encephalopathy $[1,2]$ is an acute or subacute disorder, caused by a deficiency in thiamine (vitamin B1) and usually occurs between 4 and 12 weeks postoperatively. Karapanayiotides et al. [3], however reported a 53-year-old man showing Wernicke encephalopathy after restricted dietary intake and repeated vomiting 28 years after gastric surgery, emphasizing lifelong awareness in these patients.

Current diagnostic criteria for Wernicke encephalopathy yield a classical triad of symptoms: ocular impairment, cerebellar dysfunction and confusion. One in five patients although do not manifest this typical triad of symptoms. On the other hand and according to the review by Sonal Singh and Abhay Kumar [4] atypical neurologic symptoms are common. There may be a lot of other symptoms, such as optic neuropathy, papilledema, deafness, seizures, asterixis, weakness, and sensory and motor neuropathy. Even very nonspecific symptoms, such as headache, fatigue, irritability and abdominal discomfort are also reported among the early symptoms of Wernicke encephalopathy. 
Because we are often difficult to recognize clinically, it would be useful if we could quickly measure levels of thiamine in the blood. Routine laboratory test for thiamine-esters, the erythrocyte transketolase activity assay takes too long to be used in an acute clinical setting. Jun Lu and Elizabeth Frank [5] recently developped a specific and sensitive method to measure more quickly thiaminediphosphate, which is the biologically active form of thiamine. The method consists of determining the whole blood thiamine levels by oxidizing thiamine and its phosphate esters to their corresponding thiochrome derivatives, after which thiochrome diphosphate is selectively measured by separation on an High Performance Liquid Chromatography column, followed by fluorometric detection [6-8]. This direct measurement of thiamine and its phosphate esters does not require the lengthy enzymatic dephosphorylation and has a short analysis time of 5.5 minutes. This thiamine assay becomes commercially available in most countries. In 2010, R Galvin et al. [9] wrote the EFNS guidelines and recommend that a blood sample for measurement of total thiamine should be drawn immediately before administration of thiamine and sent for HPLC analysis, whenever WE is suspected.

Patients presenting with any neurologic deficit after bariatric surgery, should be considered for Wernicke encephalopathy. We also keep in mind that also in the group of patients who underwent bariatric surgery there may be concomitant alcohol abuse.

Because the early neurologic signs of thiamine deficiency can recover completely with administration of thiamine, the acute stage seems to be a metabolic disorder.

On the other hand, Butterworth et al. [10] largely describe the focal cerebral vulnerability as a consequence of thiamine deficiency. They report that neuropathologic evaluation of brain tissue from Wernicke Encephalopathy patients reveals a clear pattern of selective damage to subcortical areas of the brain including thalamus and mammillary bodies, along with the midbrain inferior colliculus, and brainstem structures that include the vestibular nuclei and olivary. Hazell et al. [11] studied the mechanisms of neuronal cell death in Wernicke encephalopathy. They linked thiamine deficiency to the selective brain lesions seen in Wernicke encephalopathy, but additional studies are required to really explain the specific pattern of diencephalic and brainstem lesions due to thiamine deficiency [12].

Analysis of available data (indicate that WE after bariatric surgery occurs at a frequency of $2 \%$. Many studies [13,14] suggest that thiamine deficiency and its consequences often remain undiagnosed and probably the real incidence of the disease is largely underestimated.

Although the diagnosis of Wernicke encephalopathy is mainly clinical, MR imaging [15] may support the diagnosis. MR imaging is currently considered the most valuable method for confirming a diagnosis of WE, though the typical findings of MR imaging are observed in only $58 \%$ of WE patients. Typical symmetric damage of the mammillary bodies and brain paraventricular regions may permit a specific diagnosis of nonalcoholic WE. Cortical involvement in patients with nonalcoholic WE may be indicative of irreversible lesions and a poor prognosis. Characteristic brain MRI findings are hyperintense signals in the periaqueductal gray area and dorsal medial nucleus of the thalamus, but radiographic studies may be normal in some patients.

Once the diagnosis of Wernicke encephalopathy is presumptive or has been established, urgent treatment with thiamine is mandatory.
Because the pharmacological half-life of free thiamine is only 96 minutes, correct treatment requires administration of thiamine in two or three daily doses. Galvin et al. [8] studied many case reports and their guideline conclude treatment with $200 \mathrm{mg}$ thiamine IV thrice daily, has cured the disease in non-alcoholics. After bariatric surgery, administration of multivitamin pills has been recommended, but Galvin et al. [8] recommend parenteral supplementation because of frequent vomiting in these patients. The authors stipulate they do not have any evidence of an effective dosage in the setting after bariatric surgery.

\section{Conclusion}

With the ongoing global epidemic of obesity the need for obesity surgery will increase, thus leading to increased occurrence of Wernicke encephalopathy. This emphasizes the importance of awareness of this clinical entity and the need for early detection and prevention. Further studies are needed to determine the actual prevalence of this problem and to develop protocols for preventive thiamine supplementation.

Although bariatric surgery certainly improves the overall health of an obese individual, it also makes the patient more susceptible to nutrition deficiencies. In the follow-up of bariatric surgery patients, we always need to keep in mind that inadequate caloric intake, rapid weight loss, the eventual lack of adherence to nutrition supplementation and prolonged vomiting can lead to severe nutritional deficiencies. The case presented here shows that after a RYGB, a patient may develop a complex clinical entity, leading to irreversible health problems. This underscores the importance of adequate supplementation after bariatric surgery.

\section{References}

1. Shenkin A, Baines M, Fell GS, Lyon TD (2006) Vitamins and trace elements. In: Burtis CA, Ashwood ER, Bruns DE (eds.) Tietz textbook of clinical chemistry and molecular diagnostics. (4thedn), Elsevier Saunders St. Louis, USA

2. Kopelman D (2002) Disorders of Memory. Brain 125: 2152-2190.

3. Karapanayiotides T, Zaloni I, Thomaides T (2006) Delayed development of Wernicke encephalopathy after gastrectomy. Arch.neurol 63: 1026-1027.

4. Sonal Singh, Abhay Kumar (2007) Wernicke encephalopathy after obesity syrgery. A systematic Review. Neurology March 13, 68: 807-811.

5. Jun Lu, Elizabeth Frank (2008) Rapid HPLC Measurement of Thiamine and Its Phosphate Esters in Whole Blood. Clinical chemistry 54: 5901-5906.

6. Talwar D, Davidson H, Cooney J, St JO’Reilly D (2000) Vitamin B1 status assessed by direct measurement of thiamin pyrophosphate in erythrocytes or whole blood by HPLC: comparison with erythrocyte transketolase activation assay. Clin Chem 46: 704-710.

7. Lynch PL, Young IS (2000) Determination of thiamine by highperformance liquid chromatography. J Chromatogr A 881: 267-284.

8. Losa R, Sierra MI, Fernandez A, Blanco D, Buesa JM (2005) Determination of thiamine and its phosphorylated forms in human plasma, erythrocytes and urine by HPLC and fluorescence detection: a preliminary study on cancer patients. J Pharm Biomed Anal 37: 1025-1029.

9. Galvin R, Brathen G, Ivashynka A, Hillbom M, Tanasescu R, et al. (2010) EFNS guidelines for diagnosis, therapy and prevention of Wernicke encephalopathy. Eur J Neurol 17: 1408-1418.

10. Butterworth RF, Kril JJ, Harper C (1993) Thiamine-dependent enzyme changes in brain of alcoholics: relationship to Wernicke-Korsakoff syndrome. Alcohol Clin Exp Res 17: 1084-1088. 
Citation: Dobbelaere K, De Clercq S (2015) Case: Wernicke Encephalopathy after Bariatric Surgery. J Neurol Disord 3: 201. doi: 10.4172/2329-6895.1000201

Page 3 of 3

11. Hazell AS (2009) Astrocytes are a major target in thiamine deficiency and Wernicke's encephalopathy. Neurochem Int. 55: 129-135.

12. Calingasan N, Baker H, Sheu K-F, Gibson GE et al. (1995) Blood-brain abnormalities in vulnerable brain regions during thiamine deficiency. Exp Neurol 134: 64-72.

13. Aasheim ET, Bjorkman S, Sovik TT, Engström M, Hanvold SE, et al. (2009) Vitamin status after bariatric surgery: a randomized study of gastric bypass and duodenal switch. Am J Clin Nutr. 90: 15-22.
14. Aasheim ET (2008) Wernicke Encephalopathy after Bariatric Surgery: A Systematic Review, Annals of Surgery, 248: 714-720.

15. Fei GQ, Zhong C, Jin L, Wang J, Zhang Y, et al. (2008) Clinical characteristics and MR imaging features of nonalcoholic Wernicke encephalopathy. AJNR Am J Neuroradiol 29: 164-169. 